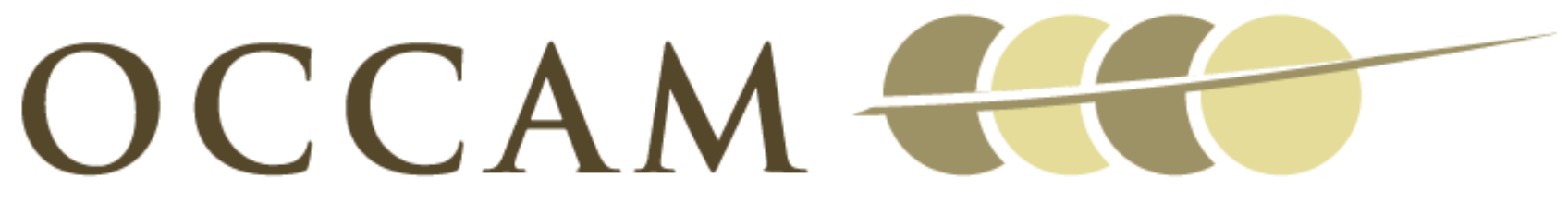

OXFORD CENTRE FOR COLLABORATIVE APPLIED MATHEMATICS

\author{
Report Number 12/10
}

Allee Effects May Slow the Spread of Parasites in a Coastal Marine Ecosystem

by

Martin Krkošek, Brendan M. Connors, Mark A. Lewis, and Robert Poulin

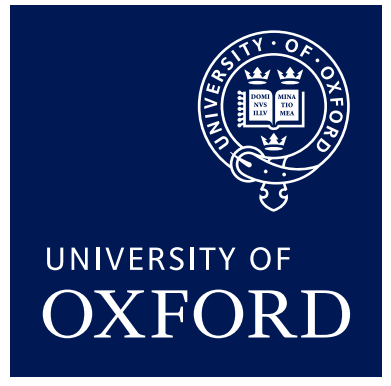

Oxford Centre for Collaborative Applied Mathematics Mathematical Institute 24 - 29 St Giles'

Oxford

OX1 3LB

England 



\title{
Allee Effects May Slow the Spread of Parasites in a Coastal Marine Ecosystem
}

\author{
Martin Krkošek, ${ }^{1,2, \star}$ Brendan M. Connors, ${ }^{2,3}$ Mark A. Lewis, ${ }^{4}$ and Robert Poulin ${ }^{1}$ \\ 1. Department of Zoology, University of Otago, Dunedin, New Zealand; 2. Salmon Coast Field Station, Simoom Sound, Canada; 3. \\ School of Resource and Environmental Management, Simon Fraser University, Burnaby, Canada; 4. Centre for Mathematical Biology, \\ Department of Mathematical and Statistical Sciences, Department of Biological Sciences, University of Alberta, Edmonton, Canada
}

Submitted August 4, 2011; Accepted November 8, 2011; Electronically published January XX, 2012

Dryad data: http://dx.doi.org/10.5061/dryad.34g16868.

Aвsтract: Allee effects are thought to mediate the dynamics of population colonization, particularly for invasive species. However, Allee effects acting on parasites have rarely been considered in the analogous process of infectious disease establishment and spread. We studied the colonization of uninfected wild juvenile Pacific salmon populations by ectoparasitic salmon lice (Lepeophtheirus salmonis) over a 4 -year period. In a data set of 68,376 fish, we observed 85 occurrences of precopular pair formation among 1,259 preadult female and 613 adult male lice. The probability of pair formation was dependent on the local abundance of lice, but this mate limitation is likely offset somewhat by mate-searching dispersal of males among host fish. A mathematical model of macroparasite population dynamics that incorporates the empirical results suggests a high likelihood of a demographic Allee effect, which can cause the colonizing parasite populations to die out. These results may provide the first empirical evidence for Allee effects in a macroparasite. Furthermore, the data give a rare detailed view of Allee effects in colonization dynamics and suggest that Allee effects may dampen the spread of parasites in a coastal marine ecosystem.

Keywords: population, dynamics, epidemiology, ocean.

\section{Introduction}

Colonization, in which a population is introduced and becomes established, is a fundamental ecological process that is rarely observed in detail. Theory predicts that colonization dynamics may be mediated by Allee effects, which, at low population size, may depress the fitness of individuals (component Allee effect) and population growth (demographic Allee effect; Allee 1931; Courchamp et al. 1999; Stephens et al. 1999). Allee effects in colonization processes can slow the spread of invasive species (Lewis and Kareiva 1993; Tobin et al. 2007) as well as the recovery of endemic species following disturbance (Kra-

\footnotetext{
* Corresponding author; e-mail: martin.krkosek@otago.ac.nz.
}

Am. Nat. 2012. Vol. 179, pp. 000-000. (C) 2012 by The University of Chicago. 0003-0147/2012/17903-53232\$15.00. All rights reserved. DOI: $10.1086 / 664458$ mer et al. 2008). The most common Allee effect in dioecious species is likely to be mate limitation, where low population density reduces the probability of male-female pair formation and subsequent reproductive success (Taylor and Hastings 2005; Gascoigne et al. 2009; Kramer et al. 2009).

Colonization is also analogous to an outbreak of infectious disease, whereby a colonizing parasite population is introduced to an uninfected host population. However, the possibility that Allee effects may dampen parasite transmission and thereby slow or prevent the spread of infectious disease has rarely been considered. The few studies that have considered Allee effects and disease spread have been theoretical, such as the emergence of Allee effects from dose-dependent establishment or infectiousness for microparasites (e.g., viruses; Dushoff 1996; Regoes et al. 2002). Models for macroparasites (e.g., worms) have considered how theoretical mating probabilities under various mating systems and parasite distributions give rise to a demographic Allee effect in the parasite population (May 1977; Anderson and May 1991; May and Woolhouse 1993; Cornell et al. 2004).

Several studies have empirically documented dose-dependent establishment, incubation time, and host mortality for microparasites (Agnew and Koella 1999; McLean and Bostock 2000; Hughes et al. 2004; Devi and Rao 2006), which are processes that may lead to a demographic Allee effect in the population dynamics of the parasite (Regoes et al. 2002). Nonetheless, for macroparasites, we are unaware of empirical examples of Allee effects, including mate limitation or other effects. However, many macroparasite taxa are dioecious and reproduce sexually, including numerous species of relevance to veterinary science and wildlife management, ranging from nematodes to ticks. Similar to the colonization dynamics of invasive or recovering species (Lewis and Kareiva 1993; Tobin et al. 2007), mate limitation could play a significant role in the establishment and spread of these kinds of parasites. 
Other mechanisms may act to decrease potential Allee effects. Dispersal, or active mate searching, is a common mechanism by which mate limitation may be overcome (Gascoigne et al. 2009). Differences between sexes in dispersal behavior arise when the benefits (and/or costs) of dispersing differ between sexes (Clobert et al. 2001). For example, in polygynous taxa, mating opportunities limit male fitness, whereas resources for offspring production and care limit female fitness, resulting in male-biased dispersal and female philopatry (Croft et al. 2003). Although well described in free-living organisms (Greenwood 1980), the same selective pressures that lead to sex-biased dispersal in these organisms are likely to also occur in sexually reproducing parasites (Hakalahti-Siren et al. 2008; Connors et al. 2011). Such behavior in parasites may limit the role of mate limitation in mediating a disease outbreak.

A demographic Allee effect may also be overcome if decreases in parasite fitness caused by mate limitation are offset by increases in other fitness components, such as parasite survival. For host-macroparasite systems, this may involve the effects of parasite pathogenicity, abundance, and aggregation on their own survival through that of their host. Increased aggregation would tend to increase the probability of male-female pair formation (May 1977) but also increase the rate of parasite-induced host mortality (Anderson and May 1978). For parasite species in which males search for females among hosts, the pathogenicity and aggregation of females may be an important determinant of reproductive success, because these factors would mediate the duration of female availability for males as well as the duration of egg production.

Using a model host-parasite system in which male-biased dispersal of adult parasites occurs (Connors et al. 2011), we combine empirical and theoretical approaches to study Allee effects in the parasite's population dynamics. The model system consists of a marine ectoparasite, the salmon louse (Lepeophtheirus salmonis), and wild pink (Oncorhynchus gorbuscha) and chum (Oncorhynchus keta) salmon in British Columbia, Canada. After entering the ocean, the uninfected juvenile salmon commence a period of exposure to lice emanating from wild and farmed sources in the marine environment (Krkošek 2010). Using a data set of 68,376 fish, we investigate density dependence and intersex differences in mating probabilities of lice; the results of these investigations are then incorporated into an Anderson-May-type model for macroparasite population dynamics (Grenfell and Dobson 1995).

\section{Life History}

Salmon lice are directly transmitted polygynous parasites that reproduce sexually while attached to a host (Pike and Wadsworth 2000). Mated adult females extrude eggstrings from which free-swimming and nonfeeding nauplii hatch, moult into copepodites, attach to a host fish, and then develop through a series of chalimus stages and then motile preadult and adult stages (Johnson and Albright 1991). Individuals in the motile stages are mobile over the surface of their host and can also move among host fish (Ritchie 1997; Connors et al. 2011). Lice feed on host surface tissues, causing morbidity and mortality at high infection intensities (Pike and Wadsworth 2000) as well as sublethal effects on physiology and behavior (Krkošek et al. 2011; Nendick et al. 2011). Mating typically occurs with the formation of a precopular pair between an adult male and a preadult female, which persists until shortly after her terminal moult, when copulation takes place (Ritchie et al. 1996; Hull et al. 1998). The male places spermatophores into the genital orifices of the female, which he then fills with sealing cement before leaving the female (Ritchie et al. 1996; Hull et al. 1998).

\section{Empirical Methods}

The data come from years 2006-2009 of a long-term monitoring program of juvenile pink and chum salmon migrating past salmon farms in the Broughton Archipelago, British Columbia, Canada (Krkošek et al. 2005b, 2006). The monitoring program conducted several surveys each spring (March-June), and for each survey we collected juvenile salmon by beach seine at $1-3-\mathrm{km}$ intervals along $\sim 100 \mathrm{~km}$ of marine migration routes (fig. 1). At each site, we retained approximately 50 pink and 50 chum salmon, usually from a single beach seine set, and nonlethally analyzed the fish to identify and enumerate sea lice on each (Krkošek et al. 2005a). Among the characteristics that we scored was the occurrence of mate guarding, in which an adult male louse had formed a precopulatory pair with a preadult female louse. We also recorded the number of preadult and adult male and female lice as well as the sea surface salinity and temperature at the site of collection.

We investigated density dependence in the probability of pair formation in two ways: (1) the probability that a focal preadult female has formed a precopulatory pair with an adult male and (2) the probability that a focal adult male has formed a precopulatory pair with a preadult female. These are both conditional probabilities in which the probability of pair formation is conditional on the presence of (1) a preadult female or (2) an adult male. Therefore, we first subsetted the data for only those fish that host (1) a preadult female or (2) an adult male. For these two scenarios, the variable representing louse density was (1) the average abundance of adult male lice per fish at the sample location or (2) the average abundance of preadult female lice per fish at the sample location. These estimates occur at the appropriate spatial scale by accom- 


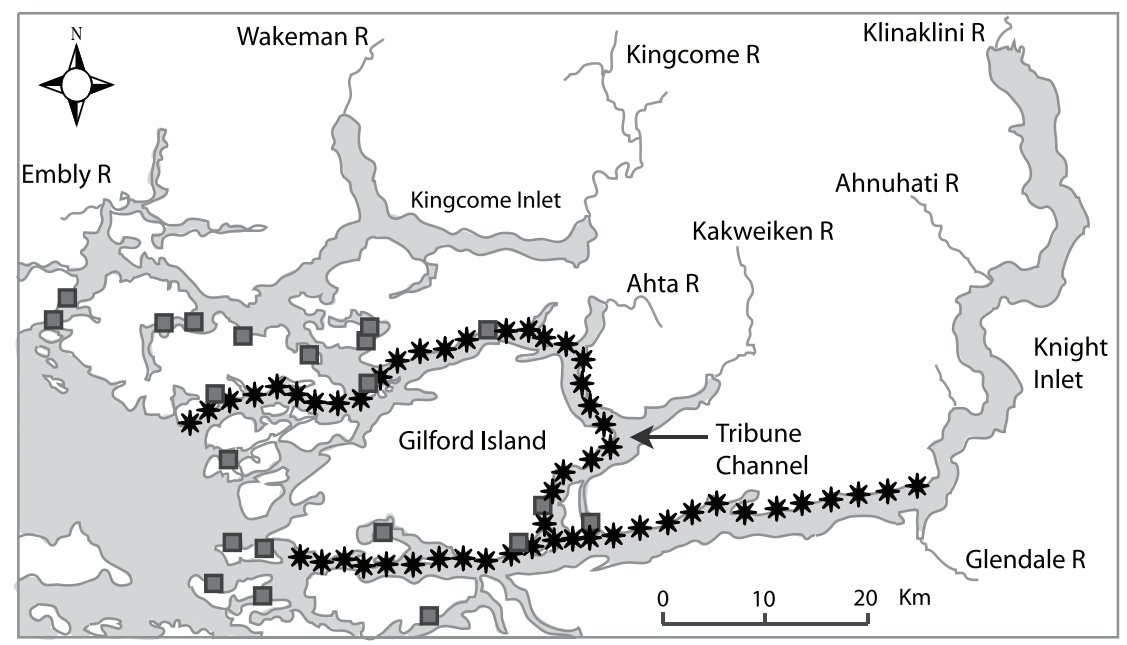

Figure 1: Map of the Broughton Archipelago showing the location of salmon farms (squares) and sample locations (stars). Sample sites study sea lice parasites on juvenile pink and chum salmon as they migrate to sea from Knight Inlet through northern and southern routes around Gilford Island.

modating the ability of motile-stage lice to swim among fish hosts (Ritchie 1997; Hull et al. 1998; Connors et al. 2008, 2011).

We used a hierarchical generalized linear model with binomial error (i.e., logistic regression) to test (1) whether the probability that a preadult female forms a precopulatory pair depends on the local average abundance of adult male lice per fish and, separately, (2) whether the probability that an adult male forms a precopulatory pair depends on the local average abundance of preadult female lice per fish. To control for nonindependence of observations from the same beach seine catch, we applied a random effect to the identity of the beach seine catch. We also considered the effects of fish body length, temperature, and salinity by including these as explanatory factors in the model. Finally, we compared the estimated probabilities of pair formation for males and females with those predicted by a Poisson distribution, which is a null model for random assortment of parasites among hosts. We used the lme 4 package and function lmer in the program $R$ for the analysis (available at http://www.R-project.org).

We further tested for evidence of aggregation of males with females via an analysis of aggregation. First, we compared Poisson and negative binomial distributions for the number of preadult females and, separately, the number of adult males among fish per collection. We assumed the aggregation parameter in the negative binomial distribution, $k$, was constant across samples. We used profile likelihood to obtain the maximum likelihood estimates and 95\% confidence intervals of $k$ for adult males $\left(k_{\mathrm{m}}\right)$ and preadult females $\left(k_{\mathrm{f}}\right)$ system-wide (lice abundances were too low to estimate $k$ for each collection). To evaluate aggregation of males with females, we used a goodnessof-fit test to test whether the frequency of presence of preadult female and adult male lice on the same fish exceeded that predicted by the corresponding preadult female-adult male bivariate negative binomial distribution as parameterized above. That is, the predicted number of observations in which a preadult female and an adult male are on the same fish in a beach seine collection is

$\hat{Y}_{i}=N_{i}\left(1-\operatorname{Pr}\left[X_{\mathrm{f}, i}=0 \mid k_{\mathrm{f}}, \bar{x}_{\mathrm{f}, i}\right]\right)\left(1-\operatorname{Pr}\left[X_{\mathrm{m}, i}=0 \mid k_{\mathrm{m}}, \bar{x}_{\mathrm{m}, i}\right]\right)$,

where $\hat{Y}_{i}$ is the predicted number of fish in collection $i$ that are infected with at least one preadult female and one adult male louse and $N_{\mathrm{i}}$ is the number of fish in collection $i$. The random variable $X_{\mathrm{f}, I}$ represents the number of preadult female lice on a fish in collection $i$ and follows a negative binomial distribution with aggregation parameter $k_{\mathrm{f}}$ estimated from the overall database and the mean $\bar{x}_{\mathrm{f}, i}$ number of preadult female lice per fish in collection $i$. Similarly for males, $X_{\mathrm{m}, I}$ is the number of adult male lice on a fish in collection $i$ and follows a negative binomial distribution with aggregation parameter $k_{\mathrm{m}}$ estimated from the overall database and mean $\bar{x}_{m, i}$ number of adult male lice per fish in collection $i$.

\section{Empirical Results}

Overall, we observed 85 occurrences of mate guarding among a total of 1,259 preadult females and 613 adult 
males in the data set, which consisted of 68,376 juvenile pink and chum salmon that averaged $48.5 \mathrm{~mm}$ in body length $(\mathrm{SD}=12.7 \mathrm{~mm})$. These data came from a total of 303 beach seine sets that contained preadult female lice for which the average abundance of adult male lice per fish in a beach seine catch ranged from 0 to 0.41 (fig. 2). The adult male lice came from 172 beach seine sets for which the average abundance of preadult female lice per fish in a beach seine catch ranged from 0 to 0.79 (fig. 2). For both preadult female lice and adult male lice, there was strong evidence that the probability of forming a precopulatory pair was dependent on the local density of the opposite sex as well as moderate-to-weak evidence that this probability was also mediated by the body length of host fish, local temperature, and local salinity (table 1).

The inclusion of a random effect for the identity of each beach seine catch did not improve the fit of the model to the data. Comparing the Akaike Information Criterion (AIC) values between the best-supported models in table 1 and the corresponding models without random effects showed that the $\triangle$ AIC values favored the generalized linear model over the generalized linear mixed model in both cases (male $\triangle \mathrm{AIC}=2$ for the mixed model; female $\Delta \mathrm{AIC}=1.8$ for the mixed model). We therefore adopted the pooled model approach and, according to a generalized linear model (i.e., a logistic regression), calculated the $95 \%$ confidence intervals on the parameter estimates. These results indicate that the effect of mate abundance on mating probability was different between sexes (table 2): for any probability, $x$, that a louse forms a precopulatory pair, there are fewer adult male lice needed to produce this probability for a focal preadult female than the abundance of preadult females needed to produce this probability for a focal adult male.

To further compare results between males and females, we used the parameter estimates from the full model in table 1 (model 8) and assumed a fish length of $50 \mathrm{~mm}$ and marine conditions of $32 \%$ salinity and $10^{\circ} \mathrm{C}$. We then compared the abundances of mates needed to produce a probability of 0.5 that a focal louse has formed a precopulatory pair. We used this reference point because it is estimable and comparable, whereas abundances required to produce $100 \%$ probability of mating are, by definition, infinite under the logistic regression model. For adult males, the average local abundance of preadult female lice per fish needed to produce a precopulatory pair at probability 0.5 was 1.03 . For preadult females, the average abundance of adult males per fish needed to form a precopulatory pair at probability 0.5 was 0.52 .

Both preadult females and adult males conformed to a negative binomial distribution, but aggregation was more pronounced for adult males than for preadult females (table 3; note that the parameter $k$ is inversely proportional
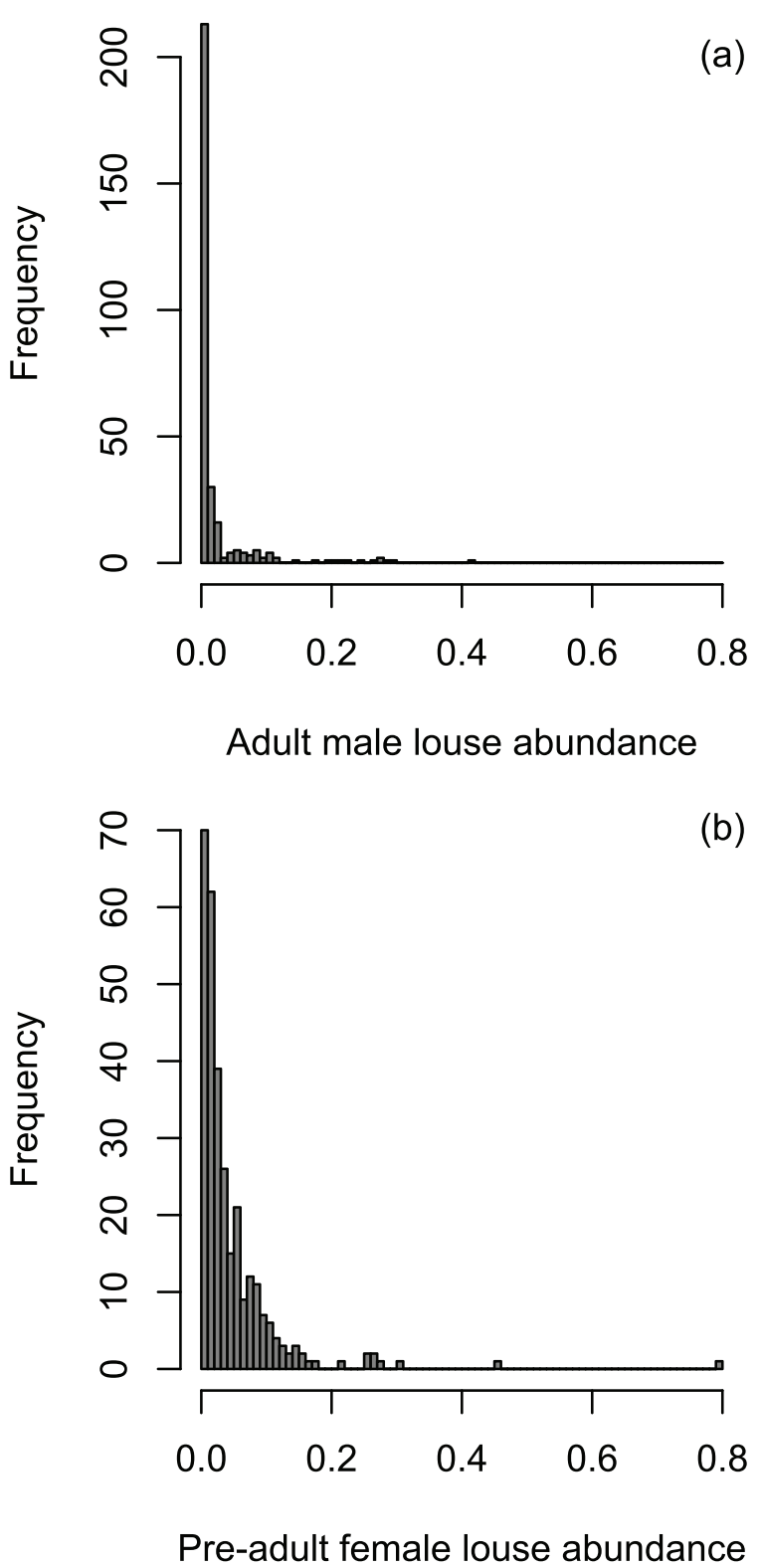

Figure 2: Frequency distribution of the average abundances of adult male lice per fish $(a)$ and preadult female lice per fish $(b)$ for each beach seine catch in the data set.

to the degree of aggregation). There were also differences in aggregation among host species; lice were more aggregated on chum than on pink salmon (table 3). To test for aggregation of males with females, we used the pooled pink and chum estimates for the negative binomial distribution, because these species form mixed-species schools within which the parasites can disperse among fish. 
Table 1: Model selection statistics for the hierarchical generalized linear model with binomial error for the probability that a preadult female or an adult male has formed a precopulatory pair

\begin{tabular}{llll}
\hline Focal sex, model & NLL & AIC & $\Delta$ AIC \\
\hline Preadult females: & & & \\
Null & 154.53 & 313.06 & 36.45 \\
AM & 139.27 & 284.53 & 7.92 \\
AM+S & 137.71 & 283.42 & 6.81 \\
AM+T & 136.99 & 281.99 & 5.38 \\
AM+L & 138.77 & 285.53 & 8.92 \\
AM+S+T & 134.65 & 279.29 & 2.68 \\
AM+S+L & 135.27 & 280.55 & 3.94 \\
AM+T+L & 136.75 & 283.5 & 6.89 \\
AM+S+T+L & 132.31 & 276.61 & 0 \\
Adult males: & & & \\
Null & 212.13 & 428.27 & 30.1 \\
PAF & 202.9 & 411.8 & 13.63 \\
PAF+S & 202.73 & 413.46 & 15.29 \\
PAF+T & 198.71 & 405.42 & 7.25 \\
PAF+L & 199.13 & 406.27 & 8.1 \\
PAF+S+T & 198.13 & 406.27 & 8.1 \\
PAF+S+L & 196.76 & 403.52 & 5.35 \\
PAF+T+L & 196.24 & 402.47 & 4.3 \\
PAF+S+T+L & 193.09 & 398.17 & 0 \\
\hline
\end{tabular}

Note: For each model, an additive random effect for the identity of a beach seine catch was placed on the intercept. AIC, Akaike Information Criterion; AM, average abundance per fish of adult males; L, length; NLL, negative log likelihood value of the model; Null, no explanatory factors; PAF, average abundance of preadult females; $\mathrm{S}$, salinity; $\mathrm{T}$, temperature; $\triangle \mathrm{AIC}, \mathrm{AIC}$ differences between the best-supported model and each other model for each focal sex.

There were 93 occurrences in the data set for which at least one preadult female and at least one adult male were observed on a fish, of which 85 had formed a mate-guarding pair. The predicted number of such occurrences, according to the bivariate negative binomial distribution, was 55.74, for which the $P$ value of the goodness-of-fit test

Table 2: Parameter estimates (Point) and 95\% confidence intervals (Lower and Higher) for the intercept (Int), mate abundance (Mates), salinity (Salt), fish length (Length), and temperature (Temp) in the logistic regression model for each focal sex

\begin{tabular}{lcrrrr}
\hline Focal sex, estimate & Int & Mates & Salt & Temp & Length \\
\hline Preadult female: & & & & & \\
$\quad$ Point & -11.144 & 7.935 & .186 & -.058 & .035 \\
$\quad$ Lower & -16.215 & 5.355 & .066 & -.123 & .005 \\
$\quad$ Higher & -6.460 & 10.588 & .317 & -.010 & .065 \\
Adult male: & & & & & \\
$\quad$ Point & -6.734 & 2.472 & .100 & -.042 & .033 \\
$\quad$ Lower & -9.996 & 1.217 & .027 & -.082 & .012 \\
$\quad$ Higher & -3.718 & 3.726 & .179 & -.009 & .055 \\
\hline
\end{tabular}

Note: Mate abundance is the average abundance of adult male lice in the beach seine collection that are available for preadult female lice (the focal sex) or the average abundance of preadult female lice in the beach seine collection that are available for adult male lice (the focal sex). (one degree of freedom) was $8.9 \times 10^{-5}$, suggesting there was significant aggregation of males with females beyond that predicted by their separate distributions.

\section{Population Model}

In this section, we develop a mathematical model to examine the implications of the empirical results for the dynamics of louse population growth. Specifically, we asked whether the component Allee effect of mate limitation during louse colonization was likely to give rise to a demographic Allee effect that suppressed population growth. A demographic Allee effect in the parasite population dynamics might not occur if the decrease in mating probability is compensated for by a change in another lifehistory parameter, such as increased survival. This could occur for parasites, because as abundance decreases, so too does the rate of host mortality induced by parasites. Other possible sources of increased survival include relief from competition among parasites or the loss of a larval louse search image in planktivores. However, these other sources seem unlikely for salmon-lice systems because of the low abundance of parasites on fish as well as in the plankton 
Table 3: Fits of the negative binomial distribution (NB) and Poisson distribution (Pois) for the distribution of adult male lice (AM) and preadult female lice (PAF) per catch when pink $(\mathrm{P})$ and chum $(\mathrm{C})$ salmon are pooled or treated separately

\begin{tabular}{lrrrrrrc}
\hline Host, lice & \multicolumn{1}{c}{$k$} & LCI & HCI & NLL-NB & NLL-Pois & $R$ & $P$ \\
\hline P and C: & & & & & & & \\
AM & .77 & .53 & 1.21 & $2,207.057$ & $2,235.452$ & 56.79 & $4.85 \times 10^{-14}$ \\
PAF & 3.14 & 1.61 & 14.5 & $4,568.893$ & $4,572.228$ & 6.67 & .0098 \\
P: & & & & & & & \\
AM & 1.59 & .89 & 3.75 & $1,292.538$ & $1,301.24$ & 17.4 & $3.02 \times 10^{-5}$ \\
PAF & 82.71 & 3.44 & NA & $2,496.829$ & $2,496.834$ & .0098 & .92 \\
C: & & & & & & & \\
AM & .61 & .34 & 1.3 & 765.352 & 777.824 & 24.94 & $5.90 \times 10^{-7}$ \\
PAF & 2.11 & .96 & 21.09 & $1,846.67$ & $1,849.135$ & 4.928 & .026 \\
\hline
\end{tabular}

Note: Shown are the negative log-likelihoods (NLL) for each distribution, the maximum likelihood estimates of the aggregation parameter $k$ in the negative binomial distribution, and the lower (LCI) and upper (UCI) $95 \%$ confidence intervals for $k$. Poisson and negative binomial distributions were compared via a likelihood ratio test where $R$ is the test statistic, which is $\chi^{2}$ distributed with one degree of freedom for each test.

relative to other plankton species. Finally, the femalebiased sex ratio estimated in the previous section is another component of the louse mating system. We assess the implications of these complexities for demographic Allee effects in louse population dynamics via a population model.

We begin with a standard Anderson-May type model for macroparasite population dynamics (Anderson and May 1991) that is modified to include the mating probability function we estimated above. The model has the form

$$
\begin{aligned}
& \frac{d \bar{P}}{d t}=\underbrace{\beta L N}_{\begin{array}{c}
\text { Larval attachment } \\
\text { to fish hosts }
\end{array}}-\underbrace{\mu_{\mathrm{P}} \bar{P}}_{\begin{array}{c}
\text { Natural mortality of } \\
\text { attached parasites }
\end{array}} \\
& -\left(\alpha+\frac{\alpha}{k} \bar{P}\right) \bar{P}, \\
& \text { Parasite mortality due to } \\
& \text { parasite-induced host mortality }
\end{aligned}
$$

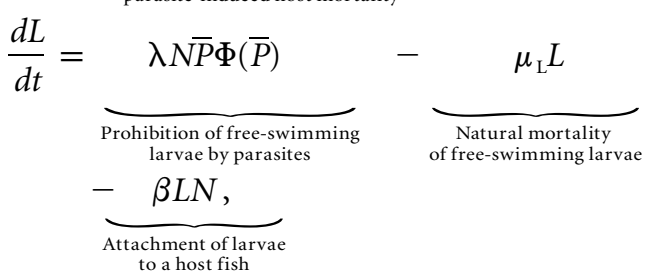

where $N$ is the abundance of the juvenile salmon population (assumed to be constant over the period of colonization), $\bar{P}$ is the average number of adult female lice per juvenile salmon, and $L$ is the abundance of free-living larvae. Parasites kill the juvenile salmon hosts at a perparasite rate $\alpha$, which affects the dynamics of the colonizing louse population (which is small) but not those of the host population (because it is large and parasites are rare). Larvae have natural death rate $\mu_{\mathrm{L}}$ and infect a host at a rate determined by the transmission coefficient $\beta$. Once attached to a fish, lice die at rate $\mu_{\mathrm{p}}$ and also die when their host dies due to a per-parasite host mortality rate $\alpha$. The average number of free-living female larvae produced per female parasite is $\lambda$. The quantity $k$ comes from the negative binomial distribution, which accounts for the aggregated nature of parasites on the host population (Shaw and Dobson 1995; Shaw et al. 1998). The term $\Phi$ is a density-dependent mating probability function, for which we use the logit function for the probability of pair formation between preadult female lice and adult male lice as estimated in the empirical results.

To analyze this model, we take an approach similar to that of Anderson and May (1991) and begin with a pseudosteady-state approximation and then analyze the dynamics relative to the net reproductive rate, $R_{0}$. Similar to Anderson and May (1991), we use the interpretation that $R_{0}$ is the average number of adult female parasite offspring produced by a mated female parasite during her lifetime when introduced into a susceptible host population. The parasite will spread in the host population if $R_{0}>1$ or die out if $R_{0}<1$. For the pseudo-steady-state approximation, we note that the duration of the free-living larval stage is short ( $\sim 5$ days) relative to the duration of the attached parasitic stage (several weeks or months; Pike and Wadsworth 2000). This implies that, all else being equal, the abundance of larval lice will quickly equilibrate and track the dynamics of the parasitic lice. We therefore let $d L / d t \rightarrow 0$, solve for $L$, and substitute $L$ into equation (2) to get

$$
\frac{d \bar{P}}{d t}=\frac{\beta \lambda N \bar{P} \Phi(\bar{P})}{\mu_{\mathrm{L}}+\beta N}-\left(\mu_{\mathrm{P}}+\alpha+\frac{\alpha}{k} \bar{P}\right) \bar{P}
$$

It is possible to evaluate the trade-off between mating 
probability and parasite longevity using equation (3). The average lifetime of an attached parasite is

$$
T=\left(\mu_{\mathrm{P}}+\alpha+\frac{\alpha}{k}\right)^{-1}
$$

which decreases as parasite abundance increases, whereas the mating probability increases with increasing parasite abundance (fig. 3). To parameterize $T$, we set $\mu_{\mathrm{p}}=$ $(30 \text { days })^{-1}$ (i.e., the life span of an adult female is $\sim 30$ days) and $\alpha=0.01 \mathrm{day}^{-1}$. These parameters are likely to vary considerably depending on abiotic factors, the size of the fish, and whether parasite-induced host mortality includes indirect effects of predation risk (Stien et al. 2005; Bricknell et al. 2006; Krkošek et al. 2006, 2009, 2011).

Parasite aggregation has an important effect on the outcome of the trade-off between parasite longevity and mating probability in producing an Allee effect: when parasite aggregation is small (i.e., when there are high $k$ values), the density dependence of longevity, $T$, becomes weak (fig. 3 ). At the levels of aggregation estimated for female lice, there are only marginal gains in average louse longevity as parasite abundance decreases. Over this same range of parasite abundance, the estimated logit function for mating probabilities at observed salinity and temperature conditions and average fish size shows a sharp decrease in mating probability as the average abundance of preadult females decreases (fig. 3; assuming a 2:1 sex ratio of females to males as indicated in the empirical results).

The analysis thus far suggests that we should expect a demographic Allee effect in sea lice population dynamics. To study these dynamics, we follow the approach of Anderson and May (1991) and look for unstable equilibria in the parasite population dynamics, conditional on local host population size and in relation to the net reproductive rate, $R_{0}$. The expression for $R_{0}$ that comes from equation (3) is

$$
R_{0}=\frac{\lambda}{\mu_{p}+\alpha} \frac{\beta N}{\mu_{L}+\beta N},
$$

where the first term is the lifetime reproductive output of larvae by a mated female parasite and the second term is the probability that a larva will attach to a host. Substitution of equation (5) into equation (3) gives

$$
\frac{d \bar{P}}{d t}=\left[\left(R_{0} \Phi-1\right)\left(\mu_{\mathrm{p}}+\alpha+\frac{\alpha}{k} \bar{P}\right)\right] \bar{P}
$$

as our model for the dynamics of the colonizing louse population.

For the mating probability function, $\Phi$, the formulae of May (1977) are not applicable, because those functions did not consider the mate-searching dispersal behavior of male parasites. For our purposes, to evaluate the possibility
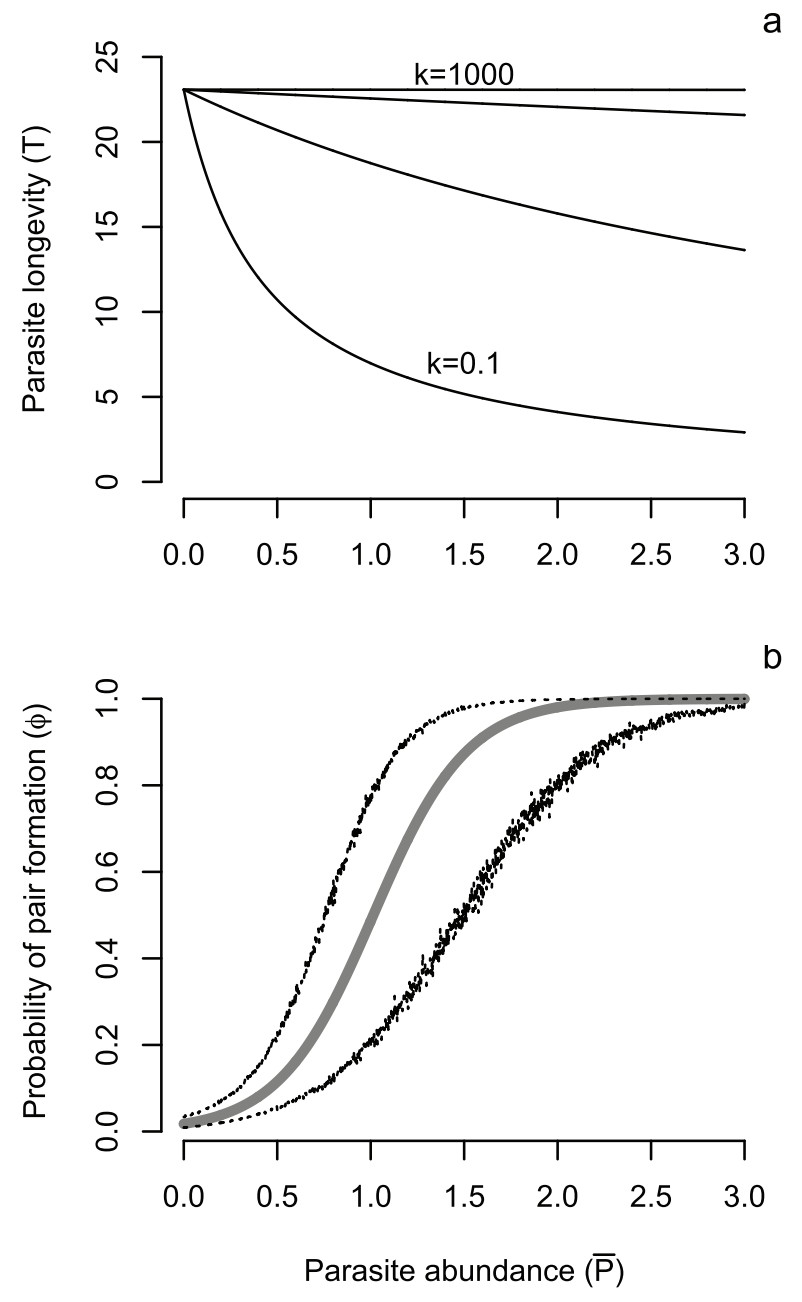

Figure 3: Density dependence of parasite longevity $(T)$ in days $(a)$ and the estimated logit function (gray line $\pm 95 \%$ confidence region) for the probability of pair formation for preadult female lice $(b)$ on fish with a body length of $50 \mathrm{~mm}$ in marine conditions of $32 \%$ salinity and $10^{\circ} \mathrm{C}$. Parasite abundance is the average number of parasites per fish. For $a$, thin lines correspond to different values of the aggregation parameter $k$ from $0.1,1,10$, and 1,000 . The confidence region in $b$ was generated by simulating parameter values for the mating probability function (eq. [7]) from a multivariate normal distribution for the parameters of the logistic regression (table 2) and their associated means and variance-covariance matrix. For each value of parasite abundance, 1,000 sets of parameter values were generated. For these, the probability of pair formation was calculated and the 0.025 and 0.975 percentiles were found.

that demographic Allee effects may occur in sea lice population dynamics due to mate limitation, we use the estimated logit function from the empirical analysis. The mating probability function, adjusted for marine conditions of $32 \%$ salinity, $10^{\circ} \mathrm{C}$, a fish length of $50 \mathrm{~mm}$, and a sex ratio of $2: 1$ for preadult females to adult males is 


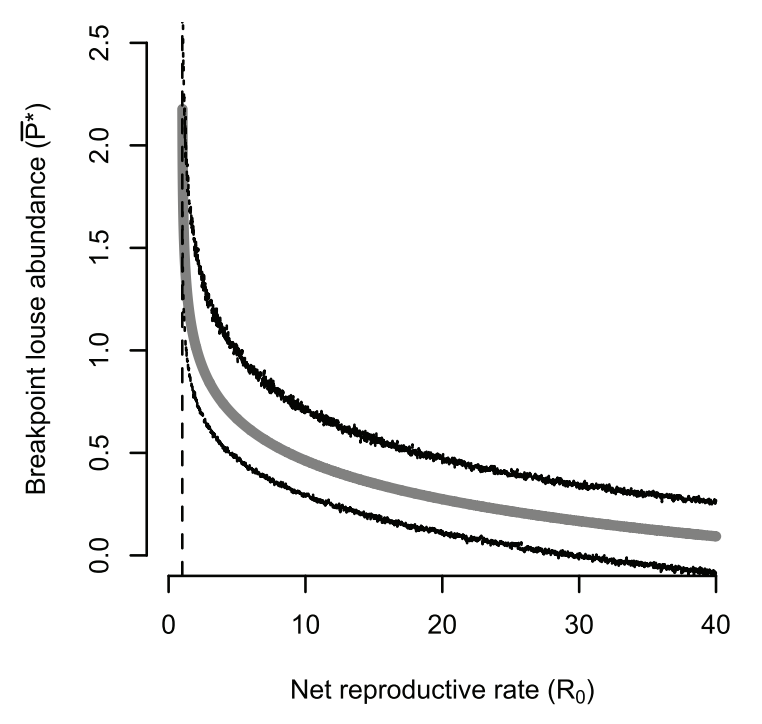

Figure 4: Estimated breakpoint in louse abundance (average number of parasites per fish; thick gray line) and $95 \%$ confidence region across the net reproductive rate $\left(R_{0}\right)$ parameter space for lice on fish with $50-\mathrm{mm}$ body length and in marine conditions of $32 \%$ salinity and $10^{\circ} \mathrm{C}$. The confidence region was generated by simulating parameter values for the mating probability function (eq. [7]) from a multivariate normal dstribution for the parameters of the logistic regression (table 2) and their associated means and variance-covariance matrix. For each value of $R_{0}, 1,000$ sets of parameter values were generated, for each of which the breakpoint louse abundance was calculated and the 0.025 and 0.975 percentiles of the breakpoint were found. The vertical dashed line marks $R_{0}=1$.

$$
\Phi(\bar{P})=\frac{\exp (a+b \bar{P})}{1+\exp (a+b \bar{P})},
$$

where $a$ captures the effects of fish size, temperature, and salinity and $b=7.9(\mathrm{SE}=1.6)$ captures density dependence, as estimated in the empirical results. To calculate an expression for the unstable equilibrium value (known as the breakpoint), we set $d \bar{P} / d t=0$ and solve for $\bar{P}^{*}$. We also simplify the model further by noting that the quadratic term in equations (2) and (3) will become very small as $\bar{P}<1$ because $\alpha<1$ and $k>1$, and so we can reasonably set $\alpha \bar{P}^{2} / k \rightarrow 0$. This gives the breakpoint abundances of lice

$$
\bar{P}^{*} \approx-\frac{1}{b}\left[a+\ln \left(R_{0}-1\right)\right],
$$

for which the predicted values and $95 \%$ confidence intervals occupy a large portion of $R_{0}$ parameter space (fig.
4). The stability analysis for the breakpoint equilibrium is given in appendix A.

The uncertainty in parameter estimates of the logistic regression for pair formation (table 2) produces a fairly narrow range of uncertainty in mating probabilities and breakpoint values across a wide region of $R_{0}$ parameter space (figs. 3, 4). However, the logistic regression also indicated that salinity and fish size also affect mating probabilities and, hence, breakpoints in louse population dynamics (table 2). In particular, breakpoint values increase with decreasing salinity and decreasing fish size (fig. 5).
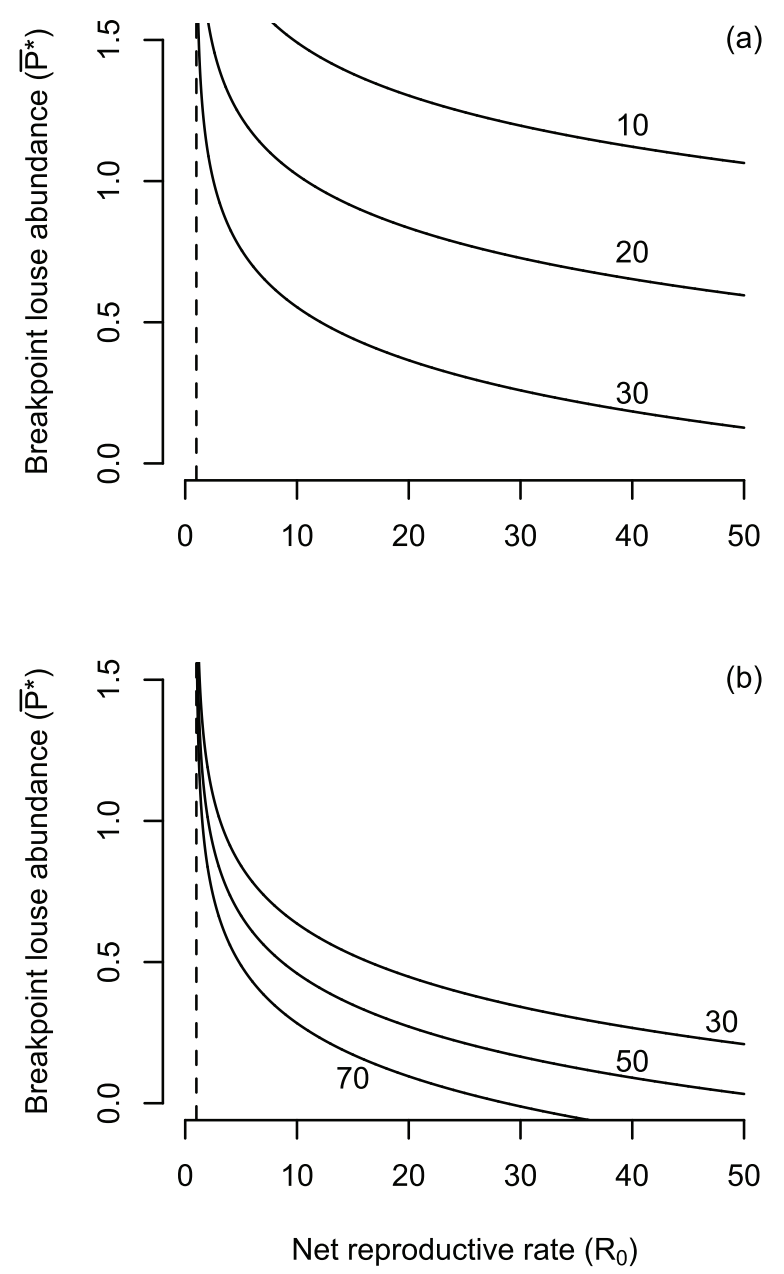

Figure 5: Breakpoint preadult female louse abundance, $\bar{P}^{*}$, over a range of plausible values for the net reproductive rate of sea lice $R_{0}$ for (a) three different conditions of salinity $(10 \%$, $20 \%$, and $30 \%$ ), as shown, and $(b)$ three different sizes of host fish (body length of 30,50 , and $70 \mathrm{~mm}$ ), as shown. Initial colonizing louse populations that are above $\bar{P}^{*}$ are expected to grow, and those below $\bar{P}^{*}$ are expected to die out. Parasite abundance is the average number of parasites per fish. The vertical dashed line marks $R_{0}=1$. 
Many other life-history parameters of lice are also likely to vary spatially and temporally because of their sensitivity to abiotic factors, such as temperature and salinity (Stien et al. 2005; Bricknell et al. 2006). Also, the value of $N$ is likely to be highly variable among years (due to variation in the number of spawners that produce the juvenile salmon and environmental conditions) as well as within years (due to mortality of juvenile salmon during their migration). Thus, $R_{0}$ is likely to also vary with $N$ as well as with other life-history parameters. However, over a wide range of plausible $R_{0}$ values, there is considerable potential for a demographic Allee effect to suppress louse population growth when colonizing a new host population (figs. 4, $5)$.

\section{Discussion}

Mate limitation is one of many potential component Allee effects that can affect the fitness of individuals at low population size (Stephens et al. 1999; Kramer et al. 2009). A demographic Allee effect is likely if the decrease in mating probability at low abundance is not offset by increases in other fitness components, such as survival. Our results provide strong empirical evidence that mate limitation occurs for salmon lice and that there is limited scope for increases in parasite survival at low parasite abundance because of parasite pathogenicity and aggregation. The theoretical results, based on an Anderson-May type macroparasite model that was modified to accommodate the empirical parameter estimates and uncertainty, suggest that a demographic Allee effect is likely to be a part of the population dynamics of salmon lice colonizing juvenile salmon.

Mate guarding was an exceptionally rare event and was observed only 85 times in 1,259 preadult females and 613 adult males among a data set consisting of 68,376 fish. The large sample size allowed us to study the colonization process in detail, but our analysis likely underestimates the overall mating success of lice. Because lice are polygynous (Hull et al. 1998), an observation of a lone male does not imply that it has not previously mated with a female on another fish. The occurrence of a lone preadult female suggests she has not previously paired with a male, because precopula persist until the female is mated shortly after her terminal moult (Ritchie et al. 1996). However, it is possible that a virgin adult female will go on to mate (Ritchie et al. 1996; Hull et al. 1998), and our data do not capture this event. Our results may therefore underestimate true mating probabilities and thereby overestimate the potential for a demographic Allee effect. However, the results do indicate that density dependence affects the rates of mate encounter.

Our analysis of patterns of overdispersion for adult male and preadult female lice indicates that males were more aggregated than females and that there may be active aggregation of males with females, as suggested in experimental work (Connors et al. 2011). If among-host movement occurs as a random process, the distribution of males among fish would be Poisson. However, if the rate parameter in a Poisson process is itself stochastic, then this can give rise to the negative binomial, which commonly characterizes parasite distributions (Shaw and Dobson 1995), and thus provides an explanation for the increased aggregation of adult males relative to females. Other passive processes, such as variation in host quality or resistance, may also lead to overdispersion but would affect both males and females and therefore cannot explain why males are more aggregated than females.

Sex-biased dispersal in search of mates is widespread throughout the animal kingdom. In general, the sex that invests the least in offspring care is the sex that disperses the most. Best described in birds and mammals (Greenwood 1980), asymmetry in reproductive investment can also select for sex-biased movement in search of mates in fish (Hutchings and Gerber 2002; Croft et al. 2003) and likely drives selection for male mate searching and female philopatry in salmon lice (Connors et al. 2011). Malebiased dispersal (de Meeus et al. 2002, 2004) as well as multiple paternity (Hasle et al. 2008) have also been detected in ectoparasitic ticks Ixodes ricinus, although the implications for Allee effects have not been investigated. Ticks, however, are vectors for several human diseases, and the movement among hosts has large implications for the relationship between biodiversity and infectious disease risk (LoGiedice et al. 2003; Keesing et al. 2010). Similar to ticks, movement of lice among hosts may have implications for the spread of other viral and bacterial marine diseases (Barker et al. 2009; Petterson et al. 2009).

The louse population model characterized the qualitative dynamics and identified the parameter space where demographic Allee effects are likely. However, many parameters are uncertain, and estimation is further complicated by interannual and seasonal variation in many factors. We therefore analyzed the model over a range of $R_{0}$ values and accounted for parameter uncertainty, which showed that there is a large region of parameter space in which demographic Allee effects could occur. The region of demographic Allee effects was sensitive to salinity, suggesting that the often estuarine conditions of coastal marine systems may further suppress parasite outbreaks. The region of demographic Allee effects was also larger on smaller host fish, suggesting that perhaps parasite-induced host mortality or motility of lice away from smaller fish may increase Allee effects on smaller hosts. However, although the model incorporated the empirical results, it did not provide a full mechanistic connection among louse 
dispersal, parasite aggregation, and mating probability, leaving scope for further theoretical development.

The conceptual framework for population colonization has useful analogies for understanding the spread of infectious disease. For example, the role of propagule pressure in the establishment of invasive species (Lockwood et al. 2005) is analogous to dose dependency in microparasite infections (McLean and Bostock 2000), in that both yield similar dynamical outcomes for population establishment and spread (Regoes et al. 2002; Taylor and Hastings 2005). We draw a similar analogy between propagule pressure and exposure to macroparasites, for which mate limitation can be an ecological mechanism mediating the spread of both invasive species and dioecious parasites. For macroparasites, our results may be the first evidence for a component Allee effect, and they suggest that a demographic Allee effect may slow the spread of infectious disease in a coastal marine ecosystem.

\section{Acknowledgments}

We are grateful to the many colleagues, technicians, and volunteers who contributed to the fieldwork: S. Durkee, H. Ford, G. Garrimone, A. Morton, E. Nelson, M. Paleczny, A. Park, S. Peacock, S. Rogers, D. Stabel, J. Volpe, and A. Yeomans-Routledge. We are also grateful to A. Ibell and S. Peacock for assembling and preparing the database. This research was supported by funding from the University of Otago, the British Columbia Pacific Salmon Forum, the David Suzuki Foundation, Watershed Watch Salmon Society, the Living Oceans Society, the Mathematics of Information Technology and Complex Systems program of the National Centres of Excellence of Canada, scholarships from the Natural Sciences and Engineering Research Council of Canada (NSERC; to M.K. and B.M.C.), and NSERC Discovery and Accelerator Grants and a Canada Research Chair (to M.A.L.). M.A.L. gratefully acknowledges a research fellowship from the Oxford Centre for Collaborative and Applied Mathematics, supported by award KUK-CI013-04 made by King Abdullah University of Science and Technology.

\section{APPENDIX}

Stability of the Breakpoint Equilibrium

To analyze the stability of the breakpoint equilibrium $\bar{P}^{*}$ (equation [8]), we linearize equation (6) about $\bar{P}^{*}$ to see whether the system will return to $\bar{P}^{*}$ if perturbed a small amount, $\rho$, away from $\bar{P}^{*}$. That is, following Wood et al. (2010), we set

$$
F(\bar{P})=\frac{d \bar{P}}{d t}=\left[\left(R_{0} \Phi(\bar{P})-1\right)\left(\mu_{\mathrm{p}}+\alpha+\frac{\alpha}{k} \bar{P}\right) \bar{P}\right.
$$

and then we calculate the derivative of $F$ with respect to $\bar{P}$ and substitute $\bar{P}^{*}$ to obtain

$$
\begin{aligned}
\Lambda=F^{\prime}\left(\bar{P}^{*}\right)= & \left(R_{0} b k\right)^{-1}\left[\left(a+\log \left(R_{0}-1\right)\right)\right. \\
& \times\left(\alpha\left(a+\log \left(R_{0}-1\right)\right)-b k\left(\alpha+\mu_{\mathrm{p}}\right)\right) \\
& \left.\times\left(R_{0}-1\right)\right]
\end{aligned}
$$

In the vicinity of $\bar{P}^{*}$, the dynamics of the small perturbation will then be governed by

$$
\frac{d \rho}{d t}=\Lambda \rho
$$

and so the equilibrium is unstable if $\Lambda>0$ and locally asymptotically stable if $\Lambda<0$ (Wood et al. 2010). For our parameter values (as indicated in the main text: $a=-$ $7.3, b=2.65, k=3.14, \mu=1 / 30, \alpha=0.01)$, the breakpoint equilibrium is unstable (fig. A1).

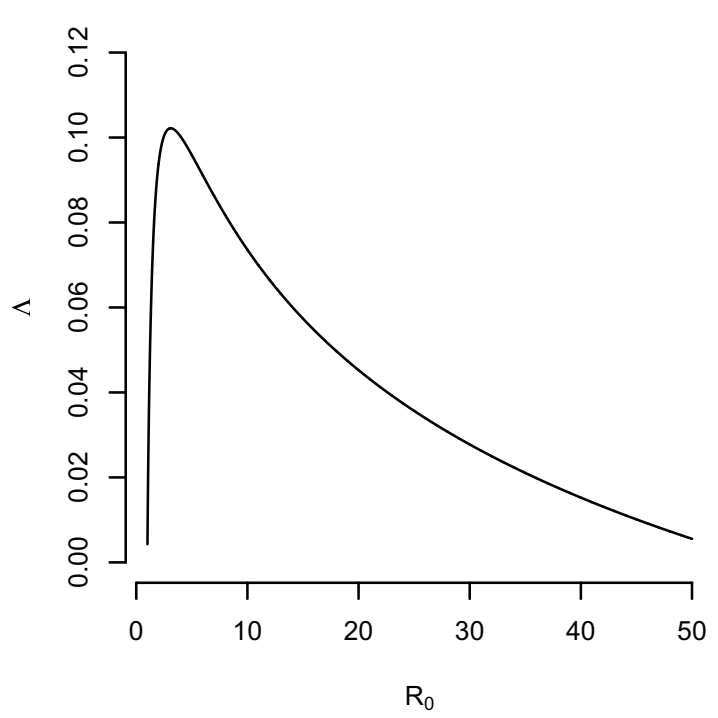

Figure A1: Eigenvalue (eq. [A2]) of the linearization (eq. [A3]) of the macroparasite population model (eq. [6]) about the breakpoint equilibrium, $\bar{P}^{*}$. 


\section{References}

Agnew, P., and J. C. Koella. 1999. Life history interactions with environmental conditions in a host-parasite relationship and the parasite's mode of transmission. Evolutionary Ecology 13:67-89.

Allee, W. C. 1931. Animal aggregations. University of Chicago Press, Chicago.

Anderson, R. M., and R. M. May. 1978. Regulation and stability of host-parasite population interactions. I. Regulatory processes. Journal of Animal Ecology 47:219-247.

- 1991. Infectious diseases of humans. Oxford University Press, Oxford.

Barker, D. E., L. M. Braden, M. P. Coombs, and B. Boyce. 2009. Preliminary studies on the isolation of bacteria from sea lice, $L e$ peophtheirus salmonis, infecting farmed salmon in British Columbia, Canada. Parasitology Research 105:1173-1177.

Bricknell, I. R., S. J. Dalesman, B. O'Shea, C. C. Pert, and A. J. M. Luntz. 2006. Effect of environmental salinity on sea lice Lepeophtheirus salmonis settlement success. Diseases of Aquatic Organisms 71:201-212.

Clobert, J., E. Danchin, A. A. Dhondt, and J. D. Nichols. 2001. Dispersal. Oxford University Press, Oxford.

Connors, B. M., M. Krkosek, and L. M. Dill. 2008. Sea lice escape predation on their host. Biology Letters 4:455-457.

Connors, B. M., C. Lagasse, and L. M. Dill. 2011. What's love got to do with it? ontogenetic drivers of dispersal in a marine ectoparasite. Behavioral Ecology 22:588-593.

Cornell, S. J., V. S. Isham, and B. T. Grenfell. 2004. Stochastic and spatial dynamics of nematode parasites in farmed ruminants. Proceedings of the Royal Society B: Biological Sciences 271:1243-1250.

Courchamp, F., T. Clutton-Brock, and B. Grenfell. 1999. Inverse density dependence and the Allee effect. Trends in Ecology \& Evolution 14:405-410.

Croft, D. P., B. Albanese, B. J. Arrowsmith, M. Botham, M. Webster, and J. Krause. 2003. Sex-biased movement in the guppy (Poecilia reticulata). Oecologia (Berlin) 137:62-68.

de Meeus, T., L. Beati, C. Delaye, A. Aeschlimann, and F. Renaud. 2002. Sex-biased genetic structure in the vector of Lyme disease, Ixodes ricinus. Evolution 56:1802-1807.

de Meeus, T., Y. Lorimier, and F. Renaud. 2004. Lyme borreliosis agents and the genetics and sex of their vector, Ixodes ricinus. Microbes and Infection 6:299-304.

Devi, K. U., and C. U. M. Rao. 2006. Allee effect in the infection dynamics of the entomopathogenic fungus Beauveria bassiana (Bals) Vuill. on the beetle, Mylabris pustulata. Mycopathologia 161: 385-394.

Dushoff, J. 1996. Incorporating immunological ideas in epidemiological models. Journal of Theoretical Biology 180:181-187.

Gascoigne, J., L. Berec, S. Gregory, and F. Courchamp. 2009. Dangerously few liaisons: a review of mate-finding Allee effects. Population Ecology 51:355-372.

Greenwood, P. J. 1980. Mating systems, philopatry and dispersal in birds and mammals. Animal Behaviour 28:1140-1162.

Grenfell, B. T., and A. P. Dobson. 1995. Ecology of infectious diseases in natural populations. Cambridge University Press, Cambridge.

Hakalahti-Siren, T., M. Bandilla, and E. T. Valtonen. 2008. Patterns of host switching in the fish ectoparasite Argulus coregoni. Behavioral Ecology and Sociobiology 62:975-982.

Hasle, G., K. H. Roed, and H. P. Leinaas. 2008. Multiple paternity in Ixodes ricinus (Acari : Ixodidae), assessed by microsatellite markers. Journal of Parasitology 94:345-347.

Hughes, W. O. H., K. S. Petersen, L. V. Ugelvig, D. Pedersen, L. Thomsen, M. Poulsen, and J. J. Boomsma. 2004. Densitydependence and within-host competition in a semelparous parasite of leaf-cutting ants. BMC Evolutionary Biology 4:45.

Hull, M. Q., A. W. Pike, A. J. Mordue, and G. H. Rae. 1998. Patterns of pair formation and mating in an ectoparasitic caligid copepod Lepeophtheirus salmonis (Kroyer 1837): implications for its sensory and mating biology. Philosophical Transactions of the Royal Society B: Biological Sciences 353:753-764.

Hutchings, J. A., and L. Gerber. 2002. Sex-biased dispersal in a salmonid fish. Proceedings of the Royal Society B: Biological Sciences 269:2487-2493.

Johnson, S. C., and L. J. Albright. 1991. The developmental stages of Lepeophtheirus salmonis (Kroyer, 1837) (Copepoda, Caligidae). Canadian Journal of Zoology 69:929-950.

Keesing, F., L. K. Belden, P. Daszak, A. Dobson, C. D. Harvell, R. D. Holt, P. Hudson, et al. 2010. Impacts of biodiversity on the emergence and transmission of infectious diseases. Nature 468:647652.

Kramer, A. M., O. Sarnelle, and R. A. Knapp. 2008. Allee effect limits colonization success of sexually reproducing zooplankton. Ecology 89:2760-2769.

Kramer, A. M., B. Dennis, A. M. Liebhold, and J. M. Drake. 2009. The evidence for Allee effects. Population Ecology 51:341-354.

Krkošek, M. 2010. Sea lice and salmon in Pacific Canada: ecology and policy. Frontiers in Ecology and the Environment 86:201-209.

Krkošek, M., A. Morton, and J. P. Volpe. 2005a. Nonlethal assessment of juvenile pink and chum salmon for parasitic sea lice infections and fish health. Transactions of the American Fisheries Society 134:711-716.

Krkošek, M., M. A. Lewis, and J. P. Volpe. 2005b. Transmission dynamics of parasitic sea lice from farm to wild salmon. Proceedings of the Royal Society B: Biological Sciences 272:689-696.

Krkošek, M., M. A. Lewis, A. Morton, L. N. Frazer, and J. P. Volpe. 2006. Epizootics of wild fish induced by farm fish. Proceedings of the National Academy of Sciences of the USA 103:15506-15510.

Krkošek, M., A. Morton, J. P. Volpe, and M. A. Lewis. 2009. Sea lice and salmon population dynamics: effects of exposure time for migratory fish. Proceedings of the Royal Society B: Biological Sciences 276:2819-2828.

Krkošek, M., B. Connors, P. Mages, S. Peacock, H. Ford, J. S. Ford, A. Morton, et al. 2011. Fish farms, parasites, and predators: implications for salmon population dynamics. Ecological Applications 21:897-914.

Lewis, M. A., and P. Kareiva. 1993. Allee dynamics and the spread of invading organisms. Theoretical Population Biology 43:141158.

Lockwood, J. L., P. Cassey, and T. Blackburn. 2005. The role of propagule pressure in explaining species invasions. Trends in Ecology \& Evolution 20:223-228.

LoGiedice, K., R. S. Ostfeld, K. A. Schmidt, and F. Keesing. 2003. The ecology of infectious disease: effects of host diversity and community composition on Lyme disease risk. Proceedings of the National Academy of Sciences of the U S A 100:567-571.

May, R. M. 1977. Togetherness among schistosomes: its effects on the dynamics of the infection. Mathematical Biosciences 35:301343 . 
May, R. M., and M. E. J. Woolhouse. 1993. Biased sex ratios and parasite mating probabilities. Parasitology 107:287-295.

McLean, A. R., and C. J. Bostock. 2000. Scrapie infections initiated at varying doses: an analysis of 117 titration experiments. Philosophical Transactions of the Royal Society B: Biological Sciences 355:1043-1050.

Nendick, L., M. Sackville, S. Tang, C. J. Brauner, and A. P. Farrell. 2011. Sea lice infection of juvenile pink salmon (Oncorhynchus gorbuscha): effects on swimming performance and postexercise ion balance. Canadian Journal of Fisheries and Aquatic Sciences 68: 241-249.

Petterson, E., M. Sandberg, and N. Santi. 2009. Salmonid alphavirus associated with Lepeophtheirus salmonis (Copepoda: Caligidae) from Atlantic salmon, Salmo salar L. Journal of Fish Diseases 32: 477-479.

Pike, A. W., and S. L. Wadsworth. 2000. Sealice on salmonids: their biology and control. Advances in Parasitology 44:233-337.

Regoes, R. R., D. Ebert, and S. Bonhoeffer. 2002. Dose-dependent infection rates of parasites produce the Allee effect in epidemiology. Proceedings of the Royal Society B: Biological Sciences 269:271279.

Ritchie, G. 1997. The host transfer ability of Lepeophtheirus salmonis (Copepoda: Caligidae) from farmed Atlantic salmon, Salmo salar L. Journal of Fish Diseases 20:153-157.

Ritchie, G., A. J. Mordue, A. W. Pike, and G. H. Rae. 1996. Observations on mating and reproductive behaviour of Lepeophtheirus salmonis, Kroyer (Copepoda: Caligidae). Journal of Experimental Marine Biology and Ecology 201:285-298.

Shaw, D. J., and A. P. Dobson. 1995. Patterns of macroparasite abundance and aggregation in wildlife populations: a quantitative review. Parasitology 111:S111-S133.

Shaw, D. J., B. T. Grenfell, and A. P. Dobson. 1998. Patterns of macroparasite aggregation in wildlife host populations. Parasitology 117:597-610.

Stephens, P. A., W. J. Sutherland, and R. P. Freckleton. 1999. What is the Allee effect? Oikos 87:185-190.

Stien, A., P. A. Bjorn, P. A. Heuch, and D. A. Elston. 2005. Population dynamics of salmon lice Lepeophtheirus salmonis on Atlantic salmon and sea trout. Marine Ecology Progress Series 290:263275.

Taylor, C. M., and A. Hastings. 2005. Allee effects in biological invasions. Ecology Letters 8:895-908.

Tobin, P. C., S. L. Whitmire, D. M. Johnson, O. N. Bjornstad, and A. M. Liebhold. 2007. Invasion speed is affected by geographical variation in the strength of Allee effects. Ecology Letters 10:3643.

Wood, C. L., K. D. Lafferty, and F. Micheli. 2010. Fishing out marine parasites? impacts of fishing on rates of parasitism in the ocean. Ecology Letters 13:761-775.

Associate Editor: Daniel Roelke Editor: Judith L. Bronstein 



\section{RECENT REPORTS}

60/11 Bayesian data assimilation in shape registration Cotter

Cotter

Vialard

61/11 Asymptotic solution of a model for bilayer organic diodes and solar cells

Richardson

Please

Kirkpatrick

62/11 Neural field model of binocular rivalry waves

Bressloff

Webber

63/11 Front propagation in stochastic neural fields

Bressloff

Webber

64/11 Stability estimates for a twisted rod under terminal loads: a threedimensional study

Majumdar

Prior

Goriely

65/11 Adaptive Finite Element Method Assisted by Stochastic Simulation of Chemical Systems

Cotter

Vejchodsky

Erban

66/11 On the shape of force-free field lines in the solar corona

Prior

Berger

$67 / 11$ Tear film thickness variations and the role of the tear meniscus

Please

Fulford

Fulford

Collins

68/11 Comment on "Frequency-dependent dispersion in porous media"

Davit

Quintard

69/11 Molecular Tilt on Monolayer-Protected Nanoparticles

Giomi

Bowick

Ma

Majumdar

70/11 The Capillary Interaction Between Two Vertical Cylinders

Cooray

Cicuta

Vella

71/11 Nonuniqueness in a minimal model for cell motility

Gallimore

Whiteley

Waters

King

Oliver

72/11 Symmetry of uniaxial global Landau-de Gennes minimizers in the

Henao theory of nematic liquid crystals

Majumdar

73/11 Filling of a Poisson trap by a population of random intermittent searchers

Bressloff

Newby

01/12 Mechanical growth and morphogenesis of seashells

Moulton

Goriely

Chirat

02/12 How linear features alter predator movement and the functional 
04/12 Numerical studies of homogenization under a fast cellular flow. Iyer

Zygalakis

05/12 Solute transport within porous biofilms: diffusion or dispersion? Davit

Byrne

Osborne

Pitt-Francis

Gavaghan

Quintard

06/12 Effects of intrinsic stochasticity on delayed reaction-diffusion patterning systems

Woolley

Baker

Gaffney

Maini

Seirin-Lee

07/12 Axial Dispersion via Shear-enhanced Diffusion in Colloidal Sus-

Griffiths pensions

Stone

08/12 Qualitative Analysis of an Integro-Differential Equation Model of

Jaina Periodic Chemotherapy

Byrne

09/12 Modeling Stem/Progenitor Cell-Induced Neovascularization and

Jain Oxygenation

Moldovan

Byrne

Copies of these, and any other OCCAM reports can be obtained from:

Oxford Centre for Collaborative Applied Mathematics

Mathematical Institute

24 - 29 St Giles'

Oxford

OX1 3LB

England

www.maths.ox.ac.uk/occam 